\title{
El comercio justo en el contexto del desarrollo sostenible
}

\section{The Fairtrade movement in the context of sustainable development}

\author{
Edison Marcelo Salas Castelo ${ }^{1}$, Carla Sofía Arguello Guadalupe ${ }^{2} \&$ Andrea Patricia Guapi \\ Auquilla ${ }^{3}$ \\ DOI: https://doi.org/10.33262/visionariodigital.v5i1.1535
}

\begin{abstract}
.
Introduction. The Fairtrade movement arises from the need for an alternative to traditional trade under the capitalist vision, which has not found a way to promote the development of nations in an equitable way. Furthermore, many authors underline the responsibility of traditional trade for the growth of inequality and the impoverishment of many nations globally. Due to this failure of traditional trade, The Fairtrade movement appears to propose a new form of commercial exchange based on respect, dialogue, equity between producers and consumers and environmental responsibility. Aim. To analyze the principles of fair trade, its principles, the obstacles to its adoption and application and the tasks to be accomplished and the articulation that exists between fair trade and the sustainable development goals proposed by the United Nations Development Program. Methods. To carry out this research work, the documentary analysis methodology was used, including relevant documents on the research subject. Different bibliographic resources were analyzed, with special emphasis on the Latin American context Results. There are many barriers for small producers to adopt and implement projects under this alternative trade scheme, such as prohibitive costs of certifications, weak representation of producers in the organizations that decide the criteria for awarding Fairtrade certifications, low level of knowledge of the citizens about Fairtrade's principles and activities, producers' lack of economic and financial resources, among others. Conclusions. Access to the Fairtrade scheme is easier for large producer organizations

\footnotetext{
${ }^{1}$ Escuela Superior Politécnica de Chimborazo, Facultad de Recursos Naturales, Carrera de Recursos Naturales

Renovables, Chimborazo, Riobamba, Ecuador. esalas@espoch.edu.ec, https://orcid.org/0000-0003-0833-8017

${ }^{2}$ Escuela Superior Politécnica de Chimborazo, Facultad de Recursos Naturales, Carrera de Turismo,

Chimborazo, Riobamba, Ecuador., c_arguello@espoch.edu.ec, https://orcid.org/0000-0003-2102-4290

${ }^{3}$ Investigadora Independiente, Chimborazo, Riobamba, Ecuador gaaplibra@yahoo.es,

https://orcid.org/0000-0003-0711-6391
} 
with greater experience in issues such as exports, with mature organizational structures, to give a few examples. This causes small producers to be increasingly marginalized. However, Fairtrade well applied is undoubtedly an advantageous tool for sustainable development.

Keywords: Sustainability, responsible consumption, equality, social responsibility

\section{Resumen.}

Introducción. El comercio justo nace de la necesidad de una alternativa al comercio tradicional desde la visión capitalista, el cual no ha encontrado la forma de impulsar el desarrollo de las naciones de manera equitativa. Mas aun, un gran número de autores subrayan la responsabilidad del comercio tradicional sobre el crecimiento de la desigualdad y el empobrecimiento de muchas naciones a nivel global. Ante este fracaso del comercio tradicional, el comercio justo surge para plantear una forma nueva de intercambio comercial basado en el respeto, el diálogo, la equidad entre productores y consumidores y la responsabilidad ambiental Objetivos. Analizar los principios del comercio justo, sus principios, los obstáculos para su adopción y aplicación y las tareas a realizar y la articulación que existe entre el comercio justo y los objetivos de desarrollo sostenible propuestos por el Programa de Naciones Unidas para el Desarrollo. Metodología. Para la realización de este trabajo de investigación se empleó la metodología de análisis documental, incluyendo documentos relevantes sobre el objeto de investigación. Diferentes recursos bibliográficos fueron analizados, con especial énfasis en el contexto latinoamericano. Resultados. Existen muchas barreras para que los pequeños productores adopten e implementen proyectos bajo este esquema de comercio alternativo, tales como costos demasiado elevados de las certificaciones, débil representación de los productores en las organizaciones que deciden los criterios para otorgar las certificaciones de comercio justo, bajo nivel de conocimiento de los ciudadanos sobre las principios y actividades del comercio justo, falta de recursos económicos y financieros de los productores, entre otros. Conclusiones. El acceso al esquema del comercio justo es más fácil para organizaciones de productores grandes, con mayor experiencia en temas como la exportación, con estructuras organizacional maduras, por poner unos pocos ejemplos, lo cual causa que pequeños productores sean cada vez más marginalizados. Sin embargo, el comercio justo bien aplicado es, sin duda, una ventajosa herramienta para el desarrollo sustentable.

\section{Palabras Clave:}

\section{Introducción.}

El comercio es quizá una de las actividades más antiguas en la historia de la humanidad. Diversas formas de comercio han existido desde la prehistoria. Esta actividad sin duda ha jugado y juega un rol esencial en el desarrollo de las sociedades humanas. A través del comercio, distintos actores pueden obtener los recursos necesarios para satisfacer las más diversas necesidades, ya sean estas de supervivencia o simplemente para complacer deseos o trivialidades. Así mismo, los actores quienes tienen excedentes de productos pueden ofrecerlos a otros individuos para así obtener los recursos necesarios que, a su vez, les permitan obtener productos de los cuales ellos carecen. Esta cadena de ofrecer y demandar productos se ha mantenido por milenios. 
Con el desarrollo de las sociedades, también el comercio experimentó un cambio. Este cambio no siempre fue positivo en términos de igualdad o ética, es más, en muchas ocasiones el comercio dio paso a relaciones perversas entre sus partes, creando así una situación en la que muchos de los actores siempre buscaron el beneficio propio o de sus familiares y asociados en desmedro de los demás causando además un deterioro dramático de la naturaleza. Estas inadecuadas relaciones comerciales han propiciado una espiral de desigualdad entre individuos y entre sociedades, fomentando a su vez la aparición de una brecha cada vez más creciente entre individuos y sociedades acaudaladas e individuos y sociedades pobres. Así, el comercio, y las empresas tradicionales son considerados cada vez más como las causantes de muchos de los problemas sociales, ambientales y económicos, ya que las empresas que operan dentro de la visión tradicional de comercio toman ventaja de la sociedad y sus inadecuadas reglas para generar beneficios sin producir valor para la comunidad (Ávila \& Campos, 2018).

La visión economicista tradicional frecuentemente sostiene que el comercio por si solo puede aliviar la pobreza y contribuir con el desarrollo sostenible (Egas, 2009). Sin embargo, por experiencia se conoce que el comercio, si no es realizado de una forma justa, tiene efectos negativos como el incremento de la pobreza e inequidad, atenta contra la provisión de alimentos en los sectores más pobres de la sociedad, pone en peligro la identidad cultural de los pueblos, y causa la destrucción de ecosistemas y pérdida de biodiversidad debido a la desmedida e irresponsable explotación de los recursos naturales (Egas, 2009).

Globalmente, se ha visto que el comercio tradicional internacional, sin ninguna duda, ha beneficiado principalmente a los países desarrollados, y como consecuencia, esta forma de comercio no ha logrado impulsar el desarrollo de los países menos favorecidos (Ferro-Soto \& Mili, 2013), provocando que los países más ricos aceleren su desarrollo a expensas de los países menos desarrollados. Más aún, parecería que las reglas del comercio internacional tradicional están diseñadas con el propósito de perpetuar esta realidad de desigualdad (Ferro-Soto \& Mili, 2013).

El comercio justo aparece debido a que es innegable que el modelo económico tradicional ha contribuido al enriquecimiento de pocos a costa del trabajo muchos, quienes, frecuentemente incluso trabajan en condiciones de explotación. El haber experimentado un orden de desarrollo basado principalmente en el modelo de crecimiento económico, fallido e injusto, ha incrementado la preocupación por alcanzar un desarrollo igualitario en el mundo. Esta preocupación se ha puesto de manifiesto por medio de la propuesta de nuevos modelos, teorías, estrategias y políticas de desarrollo. Esta realidad sugería un cambio de fondo en las relaciones comerciales para que el comercio ayude a lograr un desarrollo más equitativo de todos sus actores, dando paso al nacimiento de la iniciativa del comercio justo, junto con otros conceptos esenciales que lo cobijan y complementan.

Este artículo presenta un análisis del comercio justo, sus principios, su articulación con los objetivos del desarrollo sostenible, barreras y tareas pendientes basado en la literatura existente, principalmente de Hispanoamérica y en el Ecuador. Además, examina varios conceptos asociados 
al comercio justo. Este análisis forma parte del proyecto de diseño de un sistema de comercio justo que contribuya al mejoramiento de las condiciones económicas de los pequeños productores agrícolas del cantón Riobamba, provincia de Chimborazo, llevado a cabo en la Escuela Superior Politécnica de Chimborazo.

\section{El comercio justo}

El comercio justo es a la vez un movimiento social mundial y un sistema alternativo a las formas tradicionales de comercio (Coordinadora de Organizaciones para el Desarrollo, 2006; Coordinadora Latinoamericana y del Caribe de Pequeños(as) Productores(as) y Trabajadores(as) de Comercio Justo [CLAC], 2019) con el propósito de enmendar las inequidades sociales y económicas generadas por la economía tradicional basada en el neoliberalismo y el capitalismo (Álvarez, 2018; CLAC, 2019) desde un enfoque multidimensional, es decir considerando factores sociales, ambientales económicos y de empoderamiento ciudadano. (Coscione, 2017). Este nuevo sistema, muchas veces considerado como un instrumento de cooperación (Salvá \& Doblas, 2005), se basa en el diálogo, la transparencia y el respeto incentivando la igualdad y el acceso justo a los mercados, tanto en el comercio nacional, como en el internacional, es solidario y busca el desarrollo sostenible de los pueblos combatiendo la pobreza a través de garantizar mejores condiciones comerciales y el respeto de los derechos de los productores y trabajadores menos favorecidos o excluidos (Clark, 2017; Coordinadora de Organizaciones para el Desarrollo, 2006; Egas, 2009; World Fair Trade Organization, 2020). Este sistema alternativo de comercio, cuyos principales promotores son las organizaciones no gubernamentales (ONG) sin fines de lucro (Cayón, García, \& Pérez, 2016), propone el establecimiento de nuevas relaciones comerciales entre los países productores, que generalmente son los del sur, y los consumidores del norte, Dichas relaciones no solo buscan influir sobre el modo de intercambio de mercancías y servicios, sino que también promueven un cambio de percepción y hábitos de consumo más responsable de sus ciudadanos (Goig, 2009). Además, esta aplicación de valores como la solidaridad que debe primar en los intercambios comerciales, bajo la visión del comercio justo, deben sobrepasar las fronteras de las naciones (Goig, 2009).

Como se indica anteriormente, en la dinámica del comercio justo intervienen, entre otros, dos actores esenciales; los productores que generalmente están en el sur, en los países menos desarrollados y las organizaciones de comercio justo de los países del norte, básicamente en Estados Unidos, Canadá, Europa y en menor proporción en Japón (Salvá \& Doblas, 2005). Para efectivizar las actividades comerciales, los actores deben cumplir con ciertos requisitos fundamentales. Los productores deben garantizar que sus organizaciones operen sobre principios de democracia y que sus actividades sean social y ambientalmente sostenibles y económicamente viables. Mientras que las organizaciones operadoras de comercio justo deben garantizar a los productores: un pago justo por sus productos, el cual está compuesto de un precio mínimo más una prima adicional dependiendo del tipo de mercancía; el pago adelantado de una fracción, la cual puede variar entre el 40 y 50 por ciento, del precio final; brindar asesoría en temas de gestión y producción ambientalmente amigable, fomentando la igualdad de género, y la maximización del 
valor agregado de los productos en el origen, con el objetivo de ayudar en la generación de la mayor cantidad de empleos posibles; proporcionar información sobre de tendencias del mercado y preferencia de los consumidores, tanto actuales como potenciales (Salvá \& Doblas, 2005).

Aunque el comercio justo, como una alternativa de acceso a los mercados, nació como una respuesta a la inequidad de las relaciones comerciales internacionales, el comercio justo, sin duda, bien gestionado es una herramienta importante para el desarrollo local. Así, el comercio justo, sobre todo, se presenta como una estrategia que busca aliviar la pobreza, no exclusivamente, pero especialmente en el área rural y fomentar la generación y mejora de las capacidades locales (Fridell, 2006).

En respuesta a varios factores como la inequidad en las relaciones comerciales, unos pocos comerciantes minoristas junto con algunas organizaciones no gubernamentales dieron inicio al movimiento del comercio justo (Calisto Friant, 2016). La aparición del comercio justo se remonta al final de la década de 1940 en los Estados Unidos con la organización Ten Thousand Villages (Salvá \& Doblas, 2005). Luego de la Segunda Guerra Mundial tienen lugar los primeros actividades comerciales en base al comercio justo para romper con la dependencia económica e intercambios inequitativos de los países del norte y el sur. El objetivo era iniciar un nuevo orden en materia económica a escala internacional, para así luchar contra el proceso de empobrecimiento en el cual los países menos desarrollados estaban inmersos debido a su dependencia de los países del norte por factores económico-financieros, tecnológicos y culturales (Coscione et al., 2017). En Europa, en el Reino Unido, al final de la década de 1950, el comercio justo se abre paso con la aparición de las tiendas Oxfam, las cuales durante la siguiente década se extenderían a Holanda y Bélgica (Salvá \& Doblas, 2005). Luego de esta experiencia, en Bélgica se da inicio a la idea de las Tiendas del Mundo, también conocidas como Tiendas de la Solidaridad o Tiendas Solidarias (Salvá \& Doblas, 2005).

Ya a finales de la década de los sesenta, nace el movimiento de El Comercio Justo (Coordinadora de Organizaciones para el Desarrollo, 2006). Las tiendas que comercializaban productos de comercio justo empiezan a multiplicarse, así como organizaciones e iniciativas de cooperación dedicadas a este tipo de comercio alternativo. Más tarde a finales de los ochenta, nacen organizaciones con dedicación exclusiva y profesional en temas de comercio justo, aparecen también las primeras plataformas continentales (Coscione et al., 2017). Recién, en la primera década del siglo veintiuno se constituyen las primeras asociaciones de productores bajo la lógica del comercio justo (Coscione et al., 2017).

Es necesario indicar que para, para garantizar el origen de los productos del comercio justo y que las formas de producción de estos cumplan con los principios propuestos para esta forma alternativa de comercio se ha implementado certificados de comercio justo. Este es el caso del FLOCERT extendido por la organización Fairtrade International, a cuya red pertenecen otras organizaciones regionales como la Coordinadora Latinoamericana y del Caribe de Pequeños(as) Productores(as) y Trabajadores(as) de Comercio Justo (CLAC), Fairtrade Africa que representa a 
agricultores y trabajadores en Africa y partes de oriente medio y la Fairtrade Network of Asia \& Pacific Producers (NAPP), que opera en Asia y Oceanía (Fairtrade International, 2019).

En el caso ecuatoriano, existe una estrategia que inició con la inclusión del comercio justo en la Constitución del 2008 y la creación, en 2010, de la Dirección de Comercio Inclusivo, la cual, entre los años 2012 y 2014, se encargó del diseño de la Estrategia Ecuatoriana de Comercio Justo (Clark, 2017; Coscione et al., 2017). Además, se emitió la legislación en que se ampara el comercio justo con varios cuerpos legales aprobados como son; Ley Orgánica de Economía Popular y Solidaria (LOEPS) 2011, Código Orgánico de la Producción, Comercio e Inversiones 2010; y el Código Orgánico de Organización Territorial, Autonomías y Descentralización (COOTAD) 2010 (Clark, 2017). Para el efecto, la Ley Orgánica de Economía Solidaria manifiesta que las actividades de comercio justo son formas de organización que tienen como principios la cooperación, solidaridad y reciprocidad y que promueven el consumo ético y responsable (Clark, 2017; Coscione et al., 2017). Adicionalmente, el Plan Nacional para el Buen Vivir del 2013 incluye seis objetivos nacionales de comercio justo (Coscione et al., 2017). En junio de 2013, se crea por Decreto Ejecutivo el Ministerio de Comercio Exterior base legal y administrativa de las políticas nacionales de comercio justo, asignando a esta dependencia las competencias para generar políticas de comercio justo, comercio inclusivo y de consorcios de comercio exterior, aplicando consideraciones preferenciales para las pequeñas empresas y negocios de la economía popular y solidaria (Clark, 2017). También, en la ley, entre otras, se reconoce la responsabilidad del estado de promover el comercio justo, el consumo responsable y la de generar incentivos por responsabilidad ambiental (Clark, 2017). Finalmente, la Coordinadora Ecuatoriana de Comercio Justo (CECJ) es la responsable del desarrollo y la formulación de la Estrategia Ecuatoriana de Comercio Justo, encargada de la política pública nacional y el impulso del comercio justo (Coscione et al., 2017).

Entre las campañas impulsadas ante la necesidad de la concientización y educación sobre el comercio justo y su importancia dirigida a productores, consumidores tanto en el sector público y privado se destacan dos: Universidades Latinoamericanas por el Comercio Justo y Ciudades y Pueblos Latinoamericanos por el Comercio Justo (Coscione et al., 2017). La primera campaña tiene como objetivo la creación de una red de universidades en América Latina y el Caribe que contribuyan con el comercio justo a través de actividades de investigación, educación, extensión y vinculación en favor de organismos de pequeños productores agrícolas y artesanales, entre otros (Coscione et al., 2017). Para este fin, en el Ecuador, a esta campaña se sumaron la Universidad Tecnológica Equinoccial (UTE), de la ciudad de Quito, en el mes de mayo de 2016, y la Escuela Superior Politécnica de Chimborazo (ESPOCH), de la ciudad de Riobamba, en el mes de mayo del 2017 (CLAC, 2019; Coscione et al., 2017). En el caso de la campaña de Ciudades y Pueblos Latinoamericanos por el Comercio Justo, esta fue lanzada, en el año 2015, por la Coordinadora Latinoamericana y del Caribe de Pequeños(as) Productores(as) y Trabajadores(as) de Comercio Justo (CLAC) y la World Fair Trade Organization-Latin America (WFTO-LA). Esta campaña tiene como objetivo el generar iniciativas para la promoción de principios y valores, prácticas y 
enfoques del comercio justo a nivel local y regional (CLAC, 2019). Ciudades latinoamericanas y caribeñas como: Rio de Janeiro, Boa Esperança y Poços de Caldas, en Brasil; la comuna de la Sagrada Familia en Chile; Arroyos y Esteros en Paraguay; Marcala en Honduras; San Isidro del General y Grecia en Costa Rica; y en el Ecuador Riobamba y Quito se unieron a esta iniciativa. Riobamba fue la primera ciudad ecuatoriana en unirse, mientras que Quito se convirtió en la primera capital de un país latinoamericano en recibir esta nominación (Coscione et al., 2017).

\section{Principios del comercio justo}

El comercio justo se desarrolla dentro de un marco de principios y valores que gobiernan sus actividades. Estos principios enfatizan la necesidad de poner el bienestar de los seres humanos como aspecto fundamental y por sobre la búsqueda irresponsable de beneficios económicos. Estos principios hablan del respeto a las diferencias, la importancia del dialogo, la remuneración y ambientes de trabajo dignos, la no explotación laboral y el trabajo infantil, el respeto y protección de la naturaleza por medio de la aplicación de formas de producción y consumo responsables, entre otros los más importantes. La Tabla 1 presenta una compilación de los principios básicos del comercio justo propuestos por organizaciones que promueven este tipo alternativo de comercio y de trabajos académicos sobre el tema.

Tabla 1 Principios del comercio justo

\begin{tabular}{|c|c|}
\hline Principios & Autores \\
\hline $\begin{array}{l}\text { En las relaciones de comercio justo, los seres humanos y su } \\
\text { bienestar están por sobre la búsqueda de la maximización de } \\
\text { ganancias económicas }\end{array}$ & CLAC (2019) \\
\hline $\begin{array}{l}\text { La creación de oportunidades de acceso a mercados para productores } \\
\text { desfavorecidos basados en valores éticos, el respeto mutuo y equidad } \\
\text { entre productores y consumidores }\end{array}$ & $\begin{array}{l}\text { CLAC (2019); Coordinadora de } \\
\text { Organizaciones para el Desarrollo (2006); } \\
\text { Coscione et al. (2017); García Chiang } \\
\text { (2011); World Fair Trade Organization } \\
\text { (2017) }\end{array}$ \\
\hline $\begin{array}{l}\text { Transparencia y responsabilidad, basados en la rendición de cuentas, } \\
\text { con respecto a la administración, así como en las relaciones } \\
\text { comerciales. }\end{array}$ & $\begin{array}{l}\text { Coordinadora de Organizaciones para el } \\
\text { Desarrollo (2006); Coscione et al. (2017); } \\
\text { World Fair Trade Organization (2017) }\end{array}$ \\
\hline $\begin{array}{l}\text { Relaciones comerciales justas, estables y con contratos de largo } \\
\text { plazo, basados en el dialogo y la solidaridad }\end{array}$ & $\begin{array}{l}\text { CLAC (2019); Coordinadora de } \\
\text { Organizaciones para el Desarrollo (2006); } \\
\text { Coscione et al. (2017); García Chiang } \\
\text { (2011); World Fair Trade Organization } \\
\text { (2017) }\end{array}$ \\
\hline $\begin{array}{l}\text { Establecimiento de precios justos por medio del dialogo participativo } \\
\text { entre los actores que permita una producción sostenible, cubrir } \\
\text { costos de producción y una remuneración digna y estable que } \\
\text { permita a los productores y sus familias tener una vida digna }\end{array}$ & $\begin{array}{l}\text { CLAC (2019); Coordinadora de } \\
\text { Organizaciones para el Desarrollo (2006); } \\
\text { Coscione et al. (2017); García Chiang } \\
\text { (2011); World Fair Trade Organization } \\
\text { (2017) }\end{array}$ \\
\hline Garantizar el no trabajo infantil y la explotación laboral & $\begin{array}{l}\text { CLAC (2019); Coordinadora de } \\
\text { Organizaciones para el Desarrollo (2006); } \\
\text { Coscione et al. (2017); World Fair Trade } \\
\text { Organization (2017) }\end{array}$ \\
\hline $\begin{array}{l}\text { Rechazo a la discriminación y garantizar la equidad de género, tanto } \\
\text { en la remuneración, promoción, oportunidades laborales y libertad } \\
\text { de asociación }\end{array}$ & $\begin{array}{l}\text { CLAC (2019); Coordinadora de } \\
\text { Organizaciones para el Desarrollo (2006); }\end{array}$ \\
\hline
\end{tabular}




\begin{tabular}{|c|c|}
\hline & $\begin{array}{l}\text { Coscione et al. (2017); World Fair Trade } \\
\text { Organization (2017) }\end{array}$ \\
\hline $\begin{array}{l}\text { Proporcionar un buen ambiente laboral, seguro y saludable, en } \\
\text { condiciones dignas y de respeto a los derechos humanos, con } \\
\text { reconocimiento justo del trabajo realizado }\end{array}$ & $\begin{array}{l}\text { CLAC (2019); Coordinadora de } \\
\text { Organizaciones para el Desarrollo (2006); } \\
\text { Coscione et al. (2017); García Chiang } \\
\text { (2011); World Fair Trade Organization } \\
(2017)\end{array}$ \\
\hline $\begin{array}{l}\text { Promover y apoyar el desarrollo de capacidades, especialmente de } \\
\text { los menos favorecidos y vulnerables }\end{array}$ & $\begin{array}{l}\text { CLAC (2019); Coordinadora de } \\
\text { Organizaciones para el Desarrollo (2006); } \\
\text { Coscione et al. (2017); World Fair Trade } \\
\text { Organization (2017) }\end{array}$ \\
\hline $\begin{array}{l}\text { Promoción de los principios y objetivos del comercio justo, así como } \\
\text { de sus prácticas }\end{array}$ & $\begin{array}{l}\text { CLAC (2019); Coordinadora de } \\
\text { Organizaciones para el Desarrollo (2006); } \\
\text { Coscione et al. (2017); World Fair Trade } \\
\text { Organization (2017) }\end{array}$ \\
\hline $\begin{array}{l}\text { Promover el respeto y conservación del medio ambiente, fomentando } \\
\text { prácticas de producción responsable a lo largo de la cadena de valor }\end{array}$ & $\begin{array}{l}\text { CLAC (2019); Coordinadora de } \\
\text { Organizaciones para el Desarrollo (2006); } \\
\text { Coscione et al. (2017); García Chiang } \\
\text { (2011); World Fair Trade Organization } \\
(2017)\end{array}$ \\
\hline $\begin{array}{l}\text { Los actores involucrados juegan un papel importante en la } \\
\text { promoción de los valores y principios del comercio justo a nivel } \\
\text { local, nacional e internacional }\end{array}$ & CLAC (2019) \\
\hline $\begin{array}{l}\text { Fomentar la cultura de consumo responsable entre los ciudadanos, } \\
\text { considerando el origen y forma de producción de los bienes, } \\
\text { privilegiando aquellos productos que se puedan obtener de cadenas } \\
\text { de comercio justo y de producción sostenible }\end{array}$ & CLAC (2019); Coscione et al. (2017) \\
\hline $\begin{array}{l}\text { Generar un ambiente que favorezca el contacto directo y continuo } \\
\text { entre productores y consumidores, evitando de esta manera la acción } \\
\text { de intermediarios y especuladores, para estos no sean quienes } \\
\text { impongan los precios y así, los productores reciban un pago justo y } \\
\text { las mercancías lleguen a precios razonables a los mercados. }\end{array}$ & $\begin{array}{l}\text { García Chiang (2011); (Salvá \& Doblas, } \\
\text { 2005) }\end{array}$ \\
\hline
\end{tabular}

Elaborado por: grupo de investigación.

Juntamente con los principios del comercio justo, se debe analizar el concepto de consumo responsable, el cual es esenciales para la consecución de los objetivos que están ligados a los principios de esta forma alternativa de comercio.

\section{El comercio justo y el consumo responsable}

Para poder alcanzar varios de los objetivos que el comercio justo y los objetivos de desarrollo sostenible proponen, los individuos y las sociedades, a las cuales ellos pertenecen, deben estar conscientes de la importancia que, al igual que la producción responsable, tiene la práctica del consumo responsable. Los hábitos críticos de adquisición de mercancías que en realidad son necesarios para nuestra supervivencia y normal desarrollo de nuestras actividades, que respete el medioambiente y colabore con su conservación, se conoce como consumo responsable o sostenible (Acedo Rey, 2019). Al igual que en el comercio justo, los consumidores responsables no solo se preocupan de la calidad y el costo de los productos, sino que también se interesan sobre como estos productos fueron elaborados, la huella ecológica causado por los mismos y las condiciones 
laborales en la que operan quienes los manufacturan (Acedo Rey, 2019; Coordinadora de Organizaciones para el Desarrollo, 2006; Goig, 2009). Ya en la Cumbre de la Tierra en 2002, las Naciones Unidas manifestaron, por medio de una Declaración Oficial su preocupación por las formas insostenibles de consumo de forma particular en los países más desarrollados, haciendo un llamado a repensar y cambiar estas formas insostenibles por hábitos de consumo responsable (Coordinadora de Organizaciones para el Desarrollo, 2006). Goig (2009) expresa que, a pesar de que el consumo de masas sigue siendo el que domina los hábitos de consumo particularmente en las sociedades del mundo occidental, es evidente el surgimiento de un nuevo modelo de consumo que piensa cada vez más en la conservación ambiental, la solidaridad, la responsabilidad social y el multiculturalismo.

Como se pone de manifiesto en la Tabla 1, los principios del comercio justo demuestran las ventajas que este sistema alternativo puede potencialmente ofrecer, tanto a productores organizados del sur, como a los consumidores en diversos mercados, especialmente en aquellos de los países del norte. Estos mismos principios, luego se ven traducidos en los objetivos que el comercio justo pretende alcanzar. Estos objetivos, se encuentran íntimamente relacionados con los Objetivos del Desarrollo Sostenible propuestos por las Naciones Unidas.

\section{El comercio justo y los objetivos del desarrollo sostenible}

El desarrollo sostenible se refiere a el aprovechamiento responsable de los recursos naturales para los seres humanos, sin poner en riesgo el desarrollo de las generaciones futuras. Este concepto se sustenta sobre tres pilares fundamentales: la sostenibilidad social, la cual busca que los seres humanos vivamos en una sociedad equitativa; la sostenibilidad ambiental que nos garantice la protección y el respeto de la naturaleza para que vivir en armonía con ella en un ambiente saludable; y la viabilidad económica que brinde verdaderas oportunidades de desarrollo a los más necesitados e históricamente excluidos y que a su vez movilice los recursos necesarios para el desarrollo integral de los seres humanos y la protección ambiental.

Como guía para lograr un desarrollo sostenible a nivel global, el Programa de las Naciones Unidas para el Desarrollo (PNUD), como parte de su agenda 2030, propusieron a las naciones y habitantes del planeta 17 objetivos de desarrollo sostenible, los cuales generalmente son referidos como los ODS. Los ODS fueron adoptados por adoptados por los Estados Miembros en 2015 (Programa de las Naciones Unidas para el Desarrollo [PNUD], 2015). A continuación, se enlista los 17 ODS:

1. Fin de la pobreza

2. Hambre cero

3. Salud y bienestar

4. Educación de calidad

5. Igualdad de género

6. Agua limpia y saneamiento

7. Energía asequible y no contaminante

8. Trabajo decente y crecimiento económico

9. Industria, innovación e infraestructura 
10. Reducción de las desigualdades

11. Ciudades y comunidades sostenibles

12. Producción y consumo responsables

13. Acción por el clima

14. Vida submarina

15. Vida de ecosistemas terrestres

16. Paz, justicia e instituciones sólidas

17. Alianzas para lograr objetivos

Para lograr la consecución de estos objetivos es imprescindible la colaboración de todas las naciones y sus habitantes, especialmente de los países más desarrollados, quienes deben aceptar su responsabilidad para generar un cambio que conduzca al verdadero desarrollo de la sociedad humana en su conjunto. Es aquí donde el comercio justo puede ser una de las variadas herramientas que, si es bien gestionado, puede contribuir de manera sustantiva a la consecución de los ODS. En la Tabla 2 se muestra la articulación de los principios de comercio justo y los ODS propuesta por Comet and Carraca (2017).

Tabla 2 Articulación de los principios del comercio justo con los objetivos de desarrollo sostenible

\begin{tabular}{|c|c|}
\hline Principios del comercio justo & Objetivos y metas del desarrollo sostenible \\
\hline $\begin{array}{l}\text { Creación de oportunidades para } \\
\text { productores con desventajas } \\
\text { económicas }\end{array}$ & $\begin{array}{l}\text { - Garantizar el acceso a recursos (propiedad, control de las tierras, } \\
\text { recursos naturales, nuevas tecnologías) de las comunidades rurales } \\
\text { - } \quad \text { Movilizar recursos para luchar contra la pobreza } \\
\text { Duplicar la productividad agrícola y los ingresos de los pequeños } \\
\text { productores. }\end{array}$ \\
\hline Transparencia y responsabilidad & $\begin{array}{l}\text { - Crear instituciones eficaces, responsables y transparentes } \\
\text { - Garantizar la adopción de decisiones inclusivas, participativas y } \\
\text { representativas }\end{array}$ \\
\hline Prácticas comerciales justas & - Promover un sistema de comercio internacional equitativo \\
\hline Pago justo & $\begin{array}{l}\text { - Asegurar el acceso a una alimentación sana, nutritiva y suficiente } \\
\text { durante todo el año }\end{array}$ \\
\hline $\begin{array}{l}\text { Garantizar que no haya trabajo } \\
\text { infantil o trabajo forzoso }\end{array}$ & - Erradicación del trabajo forzoso y la explotación infantil \\
\hline $\begin{array}{l}\text { Compromiso con la no } \\
\text { discriminación, la igualdad de } \\
\text { género y el empoderamiento } \\
\text { económico de la mujer y la libertad } \\
\text { de asociación }\end{array}$ & $\begin{array}{l}\text { - Igualdad de oportunidades de liderazgo y participación plena y efectiva } \\
\text { de las mujeres en la toma de decisiones en el ámbito económico } \\
\text { - Acceso al control de tierras de las mujeres en condiciones de igualdad }\end{array}$ \\
\hline $\begin{array}{l}\text { Garantizar las buenas condiciones } \\
\text { laborales }\end{array}$ & $\begin{array}{l}\text { - Trabajo decente y equitativo para todas y todos } \\
\text { - Promover la inclusión social, económica y política evitando cualquier } \\
\text { tipo de discriminación }\end{array}$ \\
\hline $\begin{array}{l}\begin{array}{l}\text { Proporcionar el desarrollo de } \\
\text { capacidades }\end{array} \\
\end{array}$ & $\begin{array}{l}\text { - Garantizar la sostenibilidad de los sistemas de producción de alimentos } \\
\text { y aplicar prácticas agrícolas resilientes que aumenten la productividad }\end{array}$ \\
\hline Promoción del comercio justo & - Movilizar recursos financieros a países empobrecidos \\
\hline Respeto por el medioambiente & $\begin{array}{l}\text { - Mantener la diversidad genética de las semillas y las plantas cultivadas } \\
\text { mediante una buena gestión de los bancos de semillas. } \\
\text { - Resiliencia ante el cambio climático } \\
\text { - Aumentar la capacidad de gestión eficaz en relación con el cambio } \\
\text { climático. }\end{array}$ \\
\hline
\end{tabular}


Fuente: Tomado de World Fair Trade Organization (2017) y Comet and Carraca (2017)

Sin duda, el comercio puede presentar una oportunidad real para contribuir con la consecución de muchos de los objetivos de desarrollo sostenible del PNUD, sin embargo, existen todavía muchos obstáculos, que las organizaciones proponentes y aquellas que están en busca de ingresar al comercio justo deben superar.

\section{Barreras en el comercio justo}

Las barreras que existen para poder operar dentro del marco de este tipo de comercio alternativo son muy variadas, pero sin duda exigen especial atención. Estos obstáculos van desde el bajo nivel de concientización de la ciudadanía e incluso productores sobre lo que ofrece la estrategia de comercio justo, pasando por la dificultad de cubrir los costos para poder conseguir una certificación que permita a las organizaciones de pequeños productores y artesanos el acceso al comercio internacional justo, hasta la falta de políticas de diferentes gobiernos de países a nivel mundial para la promoción del comercio justo. En la Tabla 3, se presenta la compilación de varios factores que entorpecen, y en muchos casos imposibilitan a los pequeños productores el acceso a estrategias de comercio justo, lo cual causa que en muchos casos estos sean relegados y excluidos.

\section{Tabla 3 Barreras presentes en el comercio justo}

- Existe una limitada participación de los productores en los organismos que definen los criterios de certificación de sus productos

Ceccon Rocha and

Ceccon (2010)

- El acceso a y distribución de productos de comercio justo es muy reducido, estos productos generalmente se ofrecen meramente como alternativa sostenible en pocas tiendas y supermercados

- Los productos de los pequeños productores representan un bajo porcentaje de ventas comparados con productos tradicionales, los cuales continúan dominando el mercado

- Las estrategias de marketing están poco desarrolladas en este sector del comercio

- La comercialización continúa representando una limitación para los pequeños productores que no pueden conseguir una certificación, ya que, sin ella, no pueden exportar sus productos

- Bajo nivel de conocimiento de parte de la sociedad a cerca del comercio justo, sus principios y prácticas

- Falta de trabajo de concientización a la ciudadanía en el ámbito local e internacional sobre el consumo responsable que es una de las bases del comercio justo

- Oferta insuficiente, inestable y poco diversificada y por parte de pequeños productores y sus organizaciones

- Problemas con la calidad de los productos, el diseño y la presentación de los estos

- Deficiencia en el conocimiento de la elaboración de instrumentos como catálogos y otros, esenciales para el comercio exterior

- Poco conocimiento de idiomas extranjeros, lo cual presenta una desventaja en la gestión del comercio exterior

- Dificultad de acceso a capital de trabajo y financiamiento

- Bajo nivel de profesionalización y acceso a nueva tecnología, así como dificultad de acceso a asistencia técnica por parte de los pequeños productores

- Escaso nivel de compromiso de los productores con el comercio asociativo, así como con la aplicación de los principios y valores del comercio justo

- Insuficiente articulación de operaciones de comercio justo en los mercados internos y con temas de seguridad y soberanía alimentaria 
- Ausencia de políticas públicas de una gran parte de países hacia el fomento del comercio justo

- Trato inequitativo, a nivel internacional, de los productos provenientes del comercio Coscione et al. (2017) justo

- $\quad$ Los precios pagados por productos de comercio justo, así como el tamaño del mercado Fridell (2006) de este tipo de productos dependen de los gustos y exigencias de los consumidores de países en el norte

- Las organizaciones con más experiencia en la exportación resultan beneficiadas en detrimento de aquellos sin experiencia quienes terminan marginalizados

- Las dificultades logísticas, tramites y cumplimiento de requisitos dificultan el acceso al mercado de comercio justo de organizaciones pequeñas

- El costo para conseguir la certificación, por ejemplo, para una organización con menos de 500 productores, puede llegar estar alrededor de los \$6 000 la primera vez y luego unos $\$ 4000$ por año. Estos valores incluyen la solicitud inicial, una auditoria y la certificación. A eso se debe añadir los costos de adecuación de infraestructura para cumplir con los estándares. En consecuencia, organizaciones con bajos niveles económicos y de financiamiento, generalmente quedan marginados.

Elaborado por: grupo de investigación.

Todas estas dificultades que se presentan en el contexto del comercio justo, ya sea para productores u organizaciones de productores que se encuentran comercializando sus productos o para aquellos que buscan iniciar sus actividades dentro de esta estrategia comercial alternativa, deben ser abordadas con mucha atención. Hay que recalcar también que, aunque estas barreras dificultan las actividades, es innegable que la operación de dentro de este sistema de comercio puede presentar beneficios para el desarrollo de las organizaciones de productores. De ningún modo estas dificultades deben desmotivar a quienes con sus negocios y emprendimientos buscan una manera de ofrecer los frutos de su producción a través de las cadenas de comercio justo. En la siguiente sección se aborda brevemente las tareas que se tienen que llevar a cabo para promover el comercio justo y ayudar a superar las barreras que aquí han sido expuestas.

\section{Mejoras para la promoción del comercio justo}

Con el fin de proporcionar respuestas para ayudar a facilitar las actividades dentro del marco de comercio justo, varios autores presentan algunas propuestas en las que se debería trabajar para mejorar la situación de acceso al este sistema de comercio. Estas propuestas varían desde la implementación de marco jurídico en diferentes países, mejoras en la situación laboral y asistencia de los gobiernos en la generación de capacidades.

Tabla 4 presenta dichas propuestas de mejora.

\section{Propuestas}

- El comercio justo debe constar en el marco jurídico de las naciones con el fin de impulsar su desarrollo

- El comercio justo debe articularse con otras actividades solidarias como el turismo sostenible, el desarrollo local, el intercambio de saberes para incrementar su campo de acción en espacios públicos y políticos
Ceccon Rocha and Ceccon

(2010) 
ISSN: 2602-8506

- El estado y sus respectivos gobiernos den contribuir a la construcción de capacidades en las organizaciones de productores, Sin embargo, estas iniciativas tienen que venir de las organizaciones

- El comercio justo debe explorar y formular estrategias para mejorar la situación de las mujeres, los migrantes y otras personas en situación de vulnerabilidad, por medio de la inserción de estos en iniciativas productivas certificadas

Clark (2017)

Coscione et al. (2017)

- Es necesario promover de manera más decidida la formación de los pequeños productores

- Promover la expansión internacional del comercio justo por medio de campanas de promoción en información es esencial

- Se debe trabajar más en la diversificación de los productos, atrayendo y a la vez dando la oportunidad a nuevos productores, así como por medio de la formación de compromisos con productores, organizaciones, cooperativas y certificadoras

- Todas las partes involucradas en el comercio justo deben trabajar decididamente para la mejora de la eficiencia organizativa, así como la mejora de la comunicación con los ciudadanos y compradores

- Crear o mejorar los servicios de respaldo a los trabajadores del comercio justo y concientizarlos sobre las políticas de reclamos y quejas

- Establecer nuevas y mejores políticas de incentivos, a través de reformas institucionales, para promover impulsar la producción solidaria, amigable con el medioambiente, es decir, promover la producción sostenible

- Se debe resaltar la importancia del equilibrio que debe existir entre la búsqueda de la eficiencia comercial y los principios que sustentan el comercio justo

Es seguro que deben existir muchos aspectos más para mejorar y así impulsar el comercio justo, buscando garantizar que este se desarrolle cumpliendo estrictamente los principios que promulgan. Sin embargo, es necesario concentrase en los aspectos más importantes para irlos cumpliendo de manera progresiva pero concreta y efectiva.

\section{Conclusiones}

- El comercio justo es sin duda una estrategia de mercado que debe ser analizada y en lo posible implementada de manera eficaz, ya que, este tipo de comercio presenta soluciones a los problemas ocasionados por la forma tradicional de comercio.

- Se debe reflexionar sobre las instancias en las que el comercio justo en su búsqueda de posicionar sus productos en el mercado puede caer en el vicio de la competencia de manera similar que las formas de comercio capitalistas neoliberales.

- Es de vital importancia el análisis de las barreras que el acceso al comercio justo presenta, tanto a productores como a la sociedad en general es indispensable para orientar los esfuerzos hacia los temas importantes, especialmente cuando se está trabajando en el diseño de programas o estrategias de comercio justo. Así, se podrá encontrar la mejor forma de evitar o resolver las diferentes adversidades antes de que estas se presenten y estar preparados para enfrentarlas, en caso de que estas sean inevitables.

- El acceso al esquema del comercio justo es más fácil para organizaciones de productores grandes, con mayor experiencia en temas como la exportación, con estructuras organizacional maduras, por poner unos pocos ejemplos, lo cual causa que pequeños productores sean cada vez más marginalizados. 
- Es importante resaltar que casi la totalidad de la literatura analizada en este articulo hace referencia al comercio justo desde la óptica del comercio internacional y de la responsabilidad que los consumidores en países del Norte o desarrollados tienen sobre el apoyo que deben prestar a los productores del Sur o países en desarrollo a través de un pago justo por las mercancías provenientes de estos últimos. Sin embargo, la concientización de los consumidores y productores locales sobre las ventajas y responsabilidades del comercio justo no es tratada como un tema central en la literatura. Un aspecto muy importante del comercio justo, que debería impulsarse con más fuerza es el de concientizar al consumidor local sobre el trabajo que los productores realizan para proveer a los mercados de productos, el cual generalmente, muy poco apreciado. Entonces, es fundamental impulsar el comercio justo primeramente a nivel local, proveyendo a los ciudadanos locales de productos de calidad producidos bajo los principios que el comercio justo propone y luego proyectarse a la búsqueda de mercados internacionales. Es decir, el comercio justo debe empezar en casa para luego salir en búsqueda de mercados externos.

\section{Referencias Bibliográficas}

Acedo Rey, A. (2019). Consumo responsable: perfil del consumidor responsable, estrategias de marketing responsable y percepción del mercado de productos responsables.

Universidad de Sevilla, Retrieved from https://idus.us.es/bitstream/handle/11441/93399/Consumo_responsable.pdf?sequence=1 \&isAllowed $=\mathrm{y}$

Álvarez, C. S. (2018). Comercio justo y economía social y solidaria: historia y evolución de sus instituciones de fomento. Equidad y Desarrollo(30), 149-172.

Ávila, R. C., \& Campos, J. L. (2018). La economía social ante los paradigmas económicos emergentes: innovación social, economía colaborativa, economía circular, responsabilidad social empresarial, economía del bien común, empresa social y economía solidaria. [The social economy facing emerging economic concepts: social innovation, social responsibility, collaborative economy, social enterprises and solidarity economy]. CIRIEC - Espana(93), 5-50. doi:http://dx.doi.org/10.7203/CIRIEC-E.93.12901

Calisto Friant, M. (2016). Comercio justo, seguridad alimentaria y globalización: construyendo sistemas alimentarios alternativos.

Cayón, E., García, M. d. M., \& Pérez, A. (2016). El perfil del consumidor de comercio justo. Revista de Dirección y Administración de Empresas, 3, 75-95.

Ceccon Rocha, B., \& Ceccon, E. (2010). La red del Comercio Justo y sus principales actores. Investigaciones Geográficas(71), 88-101.

Clark, P. (2017). Políticas Públicas y comercio justo en el Ecuador: El caso de la Estrategia Ecuatoriana para el Comercio Justo. Obtenido de https://www. cepal. org/sites/default/files/events/files/patrick_clark__politicas_publicas_y_comercio_justo_en_el_ecuador_o.pdf. 
Comet, D., \& Carraca, A. (2017). El comercio justo y los objetivos de desarrollo sostenible. Un camino en común. La voz de los pequeños productores. Andalucía, España: IDEAS Comercio Justo, Cooperativa de interés social.

Coordinadora de Organizaciones para el Desarrollo. (2006). Comercio Justo. Retrieved from https://coordinadoraongd.org/publicaciones/monografico-comercio-justo/

Coordinadora Latinoamericana y del Caribe de Pequeños(as) Productores(as) y Trabajadores(as) de Comercio Justo. (2019). Comercio justo. Retrieved from http://claccomerciojusto.org/comercio-justo/introduccion/comercio-justo/

Coscione, M. (2017). Comercio Justo y Objetivos de Desarrollo Sostenible. In CLAC (Ed.), (Vol. 8, pp. 19).

Coscione, M., Mulder, N., Alvarez, M., Costa, S., Fuentes, A., Olmos, X., . . Vélez León, S. (2017). El aporte del comercio justo al desarrollo sostenible. Santiago de Chile y El Salvador: CEPAL y CLAC.

Egas, F. A. (2009). Comercio justo o economía solidaria. In: Afese.

Fairtrade International. (2019). Fairtrade for producers. Retrieved from http://www.fairtrade.eu/ Ferro-Soto, C., \& Mili, S. (2013). Desarrollo rural e internacionalización mediante redes de Comercio Justo del café. Un estudio del caso. Cuadernos de desarrollo rural, 10(72).

Fridell, G. (2006). Comercio justo, neoliberalismo y desarrollo rural: una evaluación histórica.

García Chiang, A. (2011). El comercio justo:¿ una alternativa de desarrollo local? Polis, 7(1), 105-140.

Goig, R. L. (2009). Consumo responsable y globalización reflexiva: un estudio referido al comercio justo en España. Revista Española del tercer sector(11), 145-165.

Programa de las Naciones Unidas para el Desarrollo. (2015). Objetivos de desarrollo sostenible. Retrieved from https://www.undp.org/content/dam/undp/library/corporate/brochure/SDGs_Booklet_Web S.Sp.pdf

Salvá, A. S., \& Doblas, N. (2005). El comercio justo: implicaciones económicas y solidarias. CIRIEC-España, revista de economía pública, social y cooperativa(51), 7-24.

Stoler, M. (2012). El justo sabor del cacao: Desafíos y ventajas del comercio justo del cacao. Universidad Andina Simón Bolívar, Sede Ecuador,

World Fair Trade Organization. (2017). Los 10 principios de comercio justo. Retrieved from The Netherlands:

https://wfto.com/sites/default/files/10\%20Fair\%20Trade\%20Principles\%20\%282017\%20 \%29_Spanish.pdf

World Fair Trade Organization. (2020). About us. Retrieved from https://wfto.com/who-weare\#definition-of-fair-trade 


\section{PARA CITAR EL ARTÍCULO INDEXADO.}

Salas Castelo, E. M., Arguello Guadalupe, C. S., \& Guapi Auquilla, A. P. (2021). El comercio justo en el contexto del desarrollo sostenible. Visionario Digital, 5(1), 36-51. https://doi.org/10.33262/visionariodigital.v5i1.1535

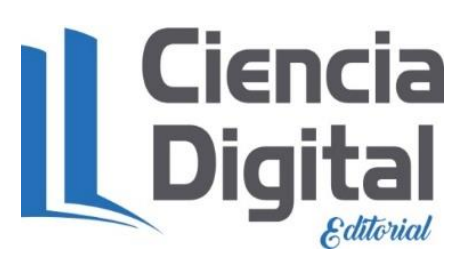

El artículo que se publica es de exclusiva responsabilidad de los autores y no necesariamente reflejan el pensamiento de la Revista Visionario Digital.

El artículo queda en propiedad de la revista y, por tanto, su publicación parcial y/o total en otro medio tiene que ser autorizado por el director de la Revista Visionario Digital.
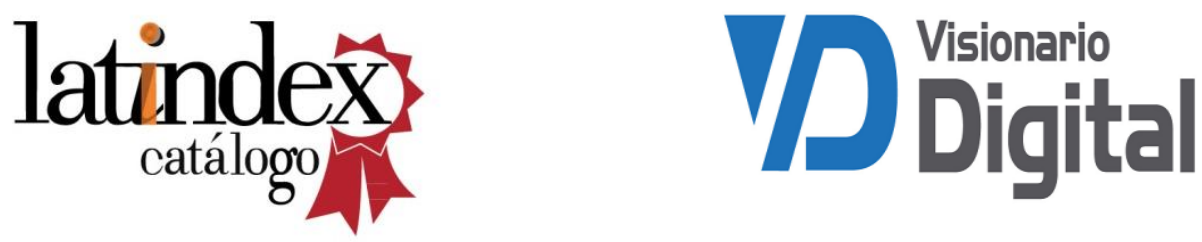